\title{
ALGEBRAIC FIBER BUNDLES
}

\author{
BY
}

STEVEN E. LANDSBURG

\begin{abstract}
When $X$ is a finite simplicial complex and $G$ is any of a certain class of groups, a classification of $G$-principal bundles over $X$ in terms of projective modules over a ring $R(G, X)$ is given. This generalizes Swan's classification of vector bundles and uses the results of Mulvey. Often, $R$ can be taken to be noetherian; in this case $\operatorname{Spec}(R)$ is usually reducible with "cohomologically trivial" irreducible components. Information is derived concerning the nature of projective modules over such rings, and some results are obtained indicating how such information reflects information about $X$.
\end{abstract}

Introduction. In 1962, Swan [11] demonstrated that the category of real or complex vector bundles over a paracompact space is equivalent via the global section functor to the category of projective modules over the ring of real- or complex-valued functions on the space. Combining a result of Mulvey [8] with some ideas based on those of Swan, we shall investigate the question of what other categories of fiber bundles can be represented "algebraically". In particular, when $X$ is a topological space and $G$ a topological group, we show in certain circumstances how to construct a noetherian scheme $\hat{X}$ and a map $X \rightarrow \hat{X}$ such that the pullback operation converts algebraic vector bundles on $\hat{X}$ into principal $G$-bundles on $X$ in such a way that some interesting information is retained. The schemes $\hat{X}$ are often affine and reducible with "cohomologically trivial" irreducible components; thus we devote $\S I I$ to the study of vector bundles over such schemes. We first show (Theorem 2.1) that the vector bundles can be classified in a way analogous to the "Mayer-Vietoris" type classification which is well known in the case in which there are only two components. We then derive several corollaries regarding what the structure of these bundles can be.

In §III we derive stable analogues of some of these results.

Finally, in §IV, we give a few applications to bundles over simplicial complexes and complex varieties and to torsion in Picard Groups.

\section{Principal bundles and projective modules.}

Definition. A ring is local if it has a unique maximal left (equivalently right) ideal. $A$ ringed space is local if each of its stalks is local.

Definition. Let $(X, \theta)$ be a ringed space. Then $\mathrm{GL}_{n} \Theta$ will denote the sheaf given by $\Gamma\left(U, \mathrm{GL}_{n} \mathcal{\theta}\right)=\mathrm{GL}_{n}(\Gamma(U, \mathcal{\theta}))$ with the obvious restriction maps.

Received by the editors August 21, 1979 and, in revised form, June 12, 1980.

AMS (MOS) subject classifications (1970). Primary 13C10, 13D15, 55F25; Secondary 16A50, $55 \mathrm{~F} 10$.

Key words and phrases. Projective module, G-principal bundle, vector bundle, affine scheme. 
Theorem 1.1 (MulVey). Let $(X, \Theta)$ be a compact local ringed space, and suppose that the center of $\Gamma(X, \theta)$ separates points (in the sense that for any $x, y \in X$, $\exists s \in \Gamma(X, \theta)$ with $s_{x}=0$ and $\left.s_{y}=1\right)$. Then the category of finitely generated projective $\Gamma(X, \mathcal{O})$-modules is equivalent to the category of locally free, locally finitely generated $\Theta$-modules.

\section{Proof. See Mulvey [8].}

REMARKS. (1) The isomorphism classes of objects in the category of locally free, locally finitely generated $\theta$-modules of rank $n$ form the pointed set $\check{H}^{1}\left(X, \mathrm{GL}_{n} \theta\right)$.

(2) Let $G$ be the group of units of a topological ring and let $\theta$ be a subsheaf of the sheaf of continuous functions from $X$ to that ring. If the theorem can be applied to $\mathcal{O}$, then it reduces the study of $G$-bundles on $X$ (where " $G$-bundle" may mean "continuous $G$-bundle", "differentiable $G$-bundle", "PL-G-bundle", "holomorphic $G$-bundle", etc., depending on $\theta$ ) to the study of algebraic line bundles on $\hat{X}=\operatorname{Spec}(\Gamma(X, \theta)) . \hat{X}$ is in general a very unwieldy construction (being nonnoetherian), and our next theorem will be aimed at correcting this situation. However, $\hat{X}$ can be both noetherian and useful, as the following example shows:

EXAMPLE 1.2. Let $X$ be a finite simplicial complex linearly embedded in general position in $\mathbf{R}^{N}$. Let $\theta$ be the sheaf given by $\Gamma(U, \theta)=\left\{f: U \rightarrow \mathbf{R}|f|_{U \cap \Delta}\right.$ is a rational function for each simplex $\Delta \subset X\}$. Then, as in the remark, we construct a noetherian scheme $(\hat{X}, \hat{\theta})$ (indeed, $\Gamma(\Delta, \hat{\theta}) \cong \mathbf{R}\left[X_{1}, \ldots, X_{n}\right]$ for each $n$-simplex $\Delta$ and $\Gamma(X, \hat{\theta})$ is a colimit of finitely many of these) such that $H^{1}\left(\hat{X}, \hat{\theta}^{*}\right) \approx$ $H^{1}\left(X, \theta^{*}\right)=H^{1}\left(X, \mathbf{R}^{*}\right)$ (here $\mathbf{R}^{*}$ is occurring as a discrete group). Note that $\mathbf{Z} / 2$ is a direct summand of $\mathbf{R}^{*}$, so that there is a surjection $H^{1}\left(X, \mathbf{R}^{*}\right) \rightarrow H^{1}(X, Z / 2)$, the latter being the group of line bundles on $X$. Thus we have constructed an affine noetherian scheme $\hat{X}$ and a continuous injection $X \stackrel{\varphi}{\rightarrow} \hat{X}$ such that every line bundle on $X$ is the pullback of an algebraic line bundle on $\hat{X}$. Later, this example will allow us to translate theorems about affine schemes into theorems about topology.

EXAMPLE 1.3. It is possible to carry out the above construction using restrictions of analytic functions rather than restrictions of polynomials, as Lonsted has shown [6]. In that case we actually get $H^{1}\left(\hat{X}, \hat{\theta}{ }^{*}\right) \approx H^{1}(X, \mathrm{Z} / 2)$, but it is quite hard to show $\hat{X}$ is noetherian. For another such construction, see Swan [12].

So far, our construction has only applied in cases where it was possible to recognize particular groups as groups of units in local rings. The following theorem will allow us to apply our methods to a much more general class of groups. First we need some definitions:

Definition. Let $\mathcal{X}$ be a category of ringed spaces. Then $\mathcal{X}$ is acceptable if for every pushout diagram

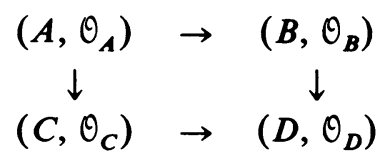


in $\mathscr{X}$, the diagram

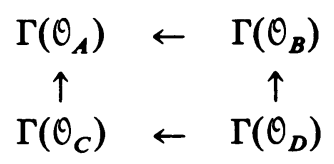

is a pullback.

EXAMPLE 1.4. If every arrow in $\mathcal{X}$ is an open inclusion, then $\mathcal{X}$ is acceptable. Suppose that $X$ is an affine scheme and that $\mathcal{X}$ is the category whose objects are intersections of irreducible components of $X$ and whose arrows are inclusions. Then if $X$ is the direct limit of $\mathcal{X}$, it follows that $\mathcal{X}$ is acceptable.

Defintion. Let $\mathcal{X}$ be any category and let $\left\{\Delta_{i} \rightarrow X\right\}_{i \in I}$ be some collection of arrows with common codomain. If $\Delta$ is the colimit of this collection (i.e., the pullback of the $\Delta_{i}$ over $X$ ), we define the union $\cup_{i \in I} \Delta_{i}$ to be the limit of the collection $\left\{\Delta \rightarrow \Delta_{i}\right\}$ (i.e., the pushout of the $\Delta_{i}$ over $\Delta$ ).

DefINITION. Let $\mathcal{X}$ be a Grothendieck topology (e.g., a category of spaces) and $\Delta \subset \mathcal{X}$ a subcategory. Then a $\Delta$-covering of an object $X \in \mathcal{X}$ is a covering $\left\{\Delta_{i}\right\}_{i \in I}$ such that:

(1) $\Delta_{i} \in \Delta, \forall i$.

(2) $\Delta_{i} \times_{X}\left(\cup_{j \in S} \Delta_{j}\right) \in \Delta$ for each subset $S \subset I$ such that $i \notin S$.

THEOREM 1.5. Let $\mathcal{X}$ be an acceptable subcategory of the category of ringed spaces which satisfy the hypotheses of Theorem 1.1. Let $\Delta$ be a subcategory of $\mathcal{X}$ such that every object in $\mathcal{X}$ has a finite $\Delta$-covering. Suppose that there exists a constant sheaf $G \subset \mathcal{O}^{*}$ such that the inclusion induces isomorphisms

$$
H^{0}(\Delta, G) \approx \Gamma\left(\Delta, \theta_{\Delta}^{*}\right) \text { for each }(\Delta, \theta) \in \Delta
$$

and

$$
H^{1}(\Delta, G) \approx H^{1}\left(\Delta, \theta_{\Delta}^{*}\right) \text { for each }(\Delta, \theta) \in \Delta .
$$

Then $H^{1}(X, G)=H^{1}\left(X, \vartheta_{X}^{*}\right)=\operatorname{Pic}\left(\Gamma\left(X, \vartheta_{X}\right)\right)$ for each $\left(X, \Theta_{X}\right) \in \mathcal{X}$.

Proof. Given an object $\left(X, \theta_{X}\right) \in \mathcal{X}$ we may write $\left(X, \theta_{X}\right)=\left(Y, \theta_{Y}\right) \cup$ $\left(\Delta, \theta_{\Delta}\right)$ where $\left(\Delta, \theta_{\Delta}\right) \in \Delta,\left(Y, \theta_{Y}\right)$ has a smaller $\Delta$-covering than $\left(X, \theta_{X}\right)$, and $\left(Y, \theta_{Y}\right) \times_{\left(X, \theta_{X}\right)}\left(\Delta, \theta_{\Delta}\right) \in \Delta$. Writing the pushout diagram

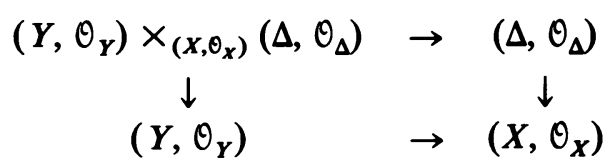

in $\mathcal{X}$, we get a pullback diagram

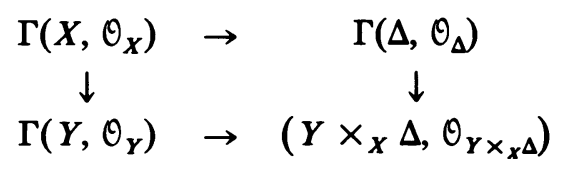


in the category of rings. This gives two Mayer-Vietoris sequences:

$$
\begin{aligned}
& H^{0}(\Delta, G) \oplus H^{0}(Y, G) \rightarrow H^{0}\left(Y \times_{X} \Delta, G\right) \rightarrow H^{1}(X, G) \\
& \Gamma\left(\Delta, \theta_{\Delta}^{*}\right) \stackrel{\oplus}{\oplus} \Gamma\left(Y, \theta_{Y}^{*}\right) \rightarrow \Gamma\left(Y \times_{X} \|, \theta_{Y}^{*} \times_{X} \Delta\right) \rightarrow \operatorname{Pic} \Gamma\left(X, \theta_{X}\right) \\
& \rightarrow \quad H^{1}(\Delta, G) \oplus H^{1}(Y, G) \quad \rightarrow \quad H^{1}\left(Y \times_{X} \Delta, G\right) \\
& \rightarrow \operatorname{Pic} \Gamma\left(\Delta, \theta_{\Delta}\right) \oplus \operatorname{Pic} \Gamma\left(Y, \theta_{Y}\right) \rightarrow \operatorname{Pic}\left(Y \times_{X} \Delta, \theta_{Y \times{ }_{X} \Delta}\right)
\end{aligned}
$$

The first, second, and fifth groups in these sequences are equal by assumption and the fourth by induction. But the latter two maps are induced by the natural transformation $H^{1}(-, G) \rightarrow H^{1}\left(-, \theta_{-}^{*}\right)=\operatorname{Pic}\left(\Gamma\left(-, \theta_{-}\right)\right.$) (the latter equality by the second remark following Theorem 1.1), and this gives an arrow $H^{1}(X, G) \rightarrow$ $\operatorname{Pic}\left(\Gamma\left(X, \theta_{X}\right)\right)$ which makes everything commute. The five-lemma completes the proof. Q.E.D.

REMARK. We have constructed an affine noetherian scheme $\hat{X}=\operatorname{Spec}\left(\Gamma\left(X, \theta_{X}\right)\right)$ and a continuous injection $X \stackrel{\psi}{\hookrightarrow} \hat{X}$ such that $\psi^{*} \theta_{\hat{X}}^{*}=G$ and such that the correspondence between $G$-principal bundles on $X$ and line bundles on $\hat{X}$ is bijective.

EXAMPLe 1.6. Let $T \subset \mathrm{C}$ be any subring such that $\operatorname{Pic}\left(T\left[X_{1}, \ldots, X_{k}\right]\right)=0$ for each $k$. (By Gauss's Lemma, this is the case when $T$ is a Dedekind ring with class group zero.) Let $\mathfrak{X}$ be the category of simplicial complexes $X$ linearly embedded in general position in $\mathbf{R}^{N}$ and endowed with the sheaf $\theta_{X}$ given by $\Gamma\left(U, \theta_{X}\right)=\{f$ : $U \rightarrow \mathbf{C} \mid$ for each simplex $\Delta,\left.f\right|_{\Delta \cap U}$ is of the form $\varphi / \psi$ where $\varphi$ and $\psi$ are polynomial functions with coefficients in $T$ \}. Then the theorem applies with $G=T^{*}$. Letting $T=\mathbf{Z}[1 / 2]$, for example, we get $H^{1}\left(X, \mathbf{Z}_{2} \times \mathbf{Z}\right)=\operatorname{Pic}\left(\Gamma\left(X, \Theta_{X}\right)\right)$ so that we have constructed a map from $X$ to an affine noetherian scheme $\hat{X}$ which induces a bijection between line bundles on $\hat{X}$ and $\mathbf{Z}_{2} \times \mathbf{Z}$-bundles on $X$. Alternatively, letting $T=Z$, we get precisely the real line bundles on $X$.

EXAMPLE 1.7. In the example above we could take $T=\mathrm{Z}[\xi]$ where $\xi$ is a primitive $n$th root of unity such that $\operatorname{Pic}(T)=0$ (for example, $n$ could be a prime $<19$-see Milnor [7]). Then the Dirichlet Unit Theorem guarantees that $\mathbf{Z} / n \mathbf{Z}$ will be a direct summand of $T^{*}$, so we have maps $\operatorname{Pic}(\hat{X}) \approx H^{1}\left(X, T^{*}\right) \rightarrow H^{1}(X, \mathbf{Z} / n \mathbf{Z})$. Thus we have constructed a map from $X$ to an affine noetherian scheme $\hat{X}$ such that every $\mathbf{Z} / n \mathbf{Z}$-bundle on $X$ is a pullback from $\hat{X}$.

In most of the above examples, the schemes we have constructed have been reducible with irreducible components which are cohomologically trivial. Our next goal is to understand the vector bundles over such schemes.

II. Vector bundles over reducible affine schemes. We now turn to the problem of classifying vector bundles over reducible affine schemes in terms of the vector bundles over the subschemes of a closed covering. The solution to this problem (Theorem 2.1) will be analyzed to provide information about the number of sections necessary to generate an arbitrary vector bundle. In §IV we will apply this information to the results of $\S$. 
Notation. In what follows, $X$ will always denote an affine reducible scheme with a closed covering $\left\{X_{1}, \ldots, X_{n}\right\}$. For example, the $X_{i}$ could be the irreducible components of $X$. As $X$ is affine, we shall write $X=\operatorname{Spec}(R)$ or $R=\Gamma(X)$, and $X_{i}=\operatorname{Spec}\left(R_{i}\right) . V B_{r}(X)$ will denote the set of rank-r vector bundles on $X$. $\mathcal{X}$ will denote the nerve of the covering $\left\{X_{i}\right\}$ of $X . \check{H}^{1}\left(\mathcal{X}, \mathrm{GL}_{r}\right)$ will denote cohomology with respect to the system of local coefficients which assigns to the simplex $\left\{i_{0}, i_{1}, \ldots, i_{n}\right\}$ the group $\mathrm{GL}_{r}\left(\Gamma\left(X_{i_{0}} \cap X_{i_{1}} \cap \cdots \cap X_{i_{n}}\right)\right)$.

We need two trivial algebraic lemmas:

LEMMA. Let $R$ be a ring and let $e, e^{\prime}: R^{n} \rightarrow R^{n}$ be idempotent. Then $e$ and $e^{\prime}$ have isomorphic images if and only if there are maps $f, g: R^{n} \rightarrow R^{n}$ satisfying the six (redundant) equations:

(1) $f e=f$,

(2) $e g=g$,

(3) $e^{\prime} f=f$,

(4) $g e^{\prime}=g$,

(5) $g f=e$,

(6) $f g=e^{\prime}$.

Proof. Write $P=\operatorname{im}(e), P^{\prime}=\operatorname{im}\left(e^{\prime}\right), Q=\operatorname{im}(1-e)$ and $Q^{\prime}=\operatorname{im}\left(1-e^{\prime}\right)$. Supposing that $P$ and $P^{\prime}$ are isomorphic, let $P \stackrel{f}{\rightarrow} P^{\prime} \stackrel{g}{\rightarrow} P$ be inverse isomorphisms and extend $f$ and $g$ to be zero on $Q$ and $Q^{\prime}$ to get a diagram

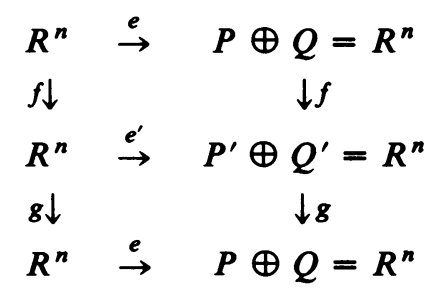

Then the facts that $f$ and $g$ are inverse isomorphisms and have been extended by zero are summarized in equations (1) through (6). Conversely, it is easy to see that if $f, g$ satisfy (1)-(6) then $\left.f\right|_{P}$ and $\left.g\right|_{P^{\prime}}$ are inverse isomorphisms. Q.E.D.

Remark. In order for $P$ to be isomorphic to $P^{\prime}$ and $Q$ to be isomorphic to $Q^{\prime}$, it is necessary and sufficient that there exist $f, g$ as above where $f=e^{\prime} u e$ and $g=e u^{-1} e^{\prime}$ for some isomorphism $u: R^{n} \rightarrow R^{n}$.

LEMMA. Let $e$ be an $m \times m$ idempotent matrix of rank $r$ over a ring $R$ and suppose that $\operatorname{im}(e)$ is free. Then there are matrices

$$
A=\left(\begin{array}{ccc}
A_{1}^{1} & \cdots & A_{m}^{1} \\
\vdots & & \vdots \\
A_{1}^{r} & \cdots & A_{m}^{r}
\end{array}\right)
$$


and

$$
B=\left(\begin{array}{ccc}
B_{1}^{1} & \cdots & B_{m}^{1} \\
\vdots & & \vdots \\
B_{1}^{r} & \cdots & B_{m}^{r}
\end{array}\right)
$$

such that $e={ }^{t} A \cdot B$ and $B \cdot{ }^{t} A$ is the $r \times r$ identity matrix. (Here ${ }^{t} A$ denotes the transpose of $A$.)

Proof. Let

$$
e^{\prime}=\left(\begin{array}{c|c}
I_{r} & 0 \\
\hline 0 & 0
\end{array}\right)
$$

and choose $f$ and $g$ as in the last lemma. Let $B$ consist of the top $r$ rows of $f$ and let ${ }^{t} A$ consist of the left $r$ columns of $g$. Q.E.D.

REMARK. More generally, in any additive category let $M=M_{1} \oplus M_{2}, e=$ the projection of $M$ on $M_{1}, N=N_{1} \oplus N_{2}$, and $e^{\prime}=$ the projection of $N$ on $N_{1}$. Then $M_{1}$ and $N_{1}$ are isomorphic if and only if there are maps

$$
M \underset{g}{\stackrel{f}{\rightleftarrows}} N
$$

such that $e=g f$ and $e^{\prime}=f g$. In this case, $f$ and $g$ can be chosen to satisfy equations (1)-(6).

TheOREM 2.1. If $X$ is the direct limit of the system of $X_{i}$ 's and their intersections, and the same is true for each finite union $X_{i_{1}} \cup \cdots \cup X_{i_{k}}$, then there is a natural long exact sequence of pointed sets

$$
1 \rightarrow \check{H}^{1}\left(\mathcal{X}, \mathrm{GL}_{r}\right) \rightarrow V B_{r}(X) \stackrel{\varphi}{\rightarrow} \bigoplus_{i=1}^{n} V B_{r}\left(X_{i}\right)
$$

These sequences commute with tensor products in the sense indicated by the diagram:

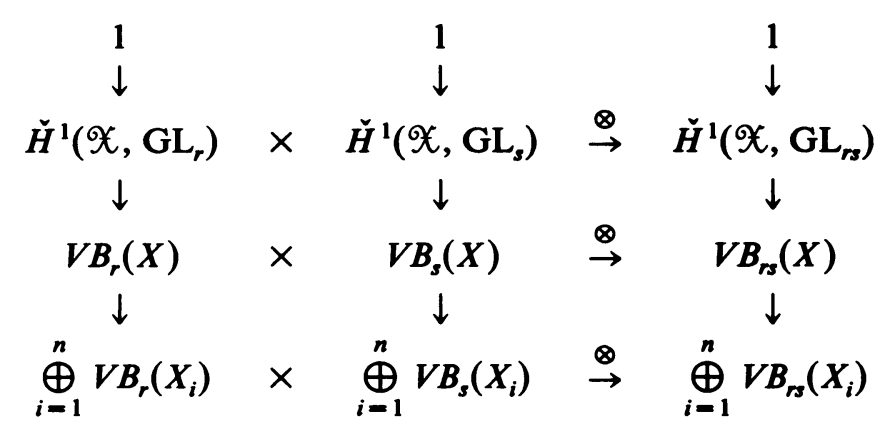

In particular, when $r=1$ the maps are group homomorphisms.

Proof. Letting $\varphi$ be the restriction map, we begin by defining a map $\operatorname{Ker}(\varphi) \stackrel{\alpha}{\rightarrow} \check{H}^{1}\left(X, \mathrm{GL}_{r}\right)$. Given $P \in \operatorname{ker}(\varphi)$, write $P \approx \operatorname{im}(e)$ where $e: R^{m} \rightarrow R^{m}$ is idempotent. (We are thinking of $P$ as a projective module over $R$.) Using the 
embedding $R \rightarrow \prod_{i=1}^{n} R_{i}$, write $e=\left(e_{1}, \ldots, e_{n}\right)$. Then, since $P \in \operatorname{ker}(\varphi), \operatorname{im}\left(e_{i}\right) \approx$ $R_{i}^{r}$. Thus we can write $e_{i}={ }^{t} A_{i} \cdot B_{i}$ where $B_{i} \cdot{ }^{t} A_{i}=I_{r}$ as in the lemma. For each $i, i^{\prime} \in\{1, \ldots, n\}$ define $p_{i i^{\prime}}$ to be the matrix $\bar{B}_{i} \cdot{ }^{t} A_{i^{\prime}}$ over $\Gamma\left(X_{i} \times_{X} X_{i^{\prime}}\right)$. (Here "bar" denotes restriction to $X_{i} \times_{X} X_{i^{\prime}}$ ) We claim that $p_{i i^{\prime}}$ is a well-defined $\mathrm{GL}_{r^{-}}$ cocycle.

First to check that $p_{i i^{\prime}}$ is invertible, note that $\overline{B_{i}} \bar{t}_{i}=\overline{e_{i}}=\overline{e_{i^{\prime}}}=\overline{B_{i^{\prime}}} \bar{t}^{t} A^{\prime}$ because $e$ has all of its entries in $R$. Thus:

But $\bar{B}_{i} \cdot{ }^{{ }^{\prime} A_{i}}=I_{r}$ so $p_{i i}$ is invertible.

$$
p_{i i^{\prime}} \overline{B_{i^{\prime}}}=\overline{B_{i}} \overline{{ }^{t} A_{i^{\prime}}} \overline{B_{i^{\prime}}}=\overline{B_{i}} \overline{{ }^{t} A_{i}} \overline{B_{i}}=\overline{B_{i}} .
$$

To see that $\left(p_{i i^{\prime}}\right)$ is a cocycle, write

$$
p_{i i^{\prime}} p_{i^{\prime} i^{\prime \prime}}=p_{i i^{\prime}} \cdot \overline{B_{i^{\prime}}} \overline{{ }^{t} A_{i^{\prime \prime}}}=\overline{B_{i}} \overline{{ }^{t} A_{i^{\prime \prime}}}=p_{i i^{*}} \text {. }
$$

Next we check that $p_{i i^{\prime}}$ is well defined: Suppose that $e$ and $\tilde{e}$ have isomorphic images (possibly $e=\tilde{e}$ ). Clearly, adding rows and columns of zeroes does not affect anything, so we may assume that $e$ and $\tilde{e}$ are of the same size, say $m \times m$. Let $f: \operatorname{im}(e) \rightarrow \operatorname{im}\left(e^{\prime}\right)$ be an isomorphism and extend $f$ by zero on the complemet of $\operatorname{im}(e)$ in $R^{n}$ so we have a commutative diagram

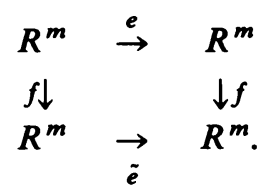

(We will write $f=\left(f_{1}, \ldots, f_{n}\right)$ ).) Suppose that $e={ }^{t} A \cdot B$ and $\tilde{e}={ }^{t} C \cdot D$, and set $p_{i i^{\prime}}=\bar{B}_{i} \cdot{ }^{t} A_{i^{\prime}}$ and $\tilde{p}_{i i^{\prime}}=\bar{D}_{i} \cdot{ }^{t} C_{i^{\prime}}$. Then

$$
\begin{aligned}
& \overline{D_{i}} \overline{f_{i}} \bar{i}^{t} A_{i} p_{i i^{\prime}}=\overline{D_{i}} \overline{f_{i}} \bar{t}_{A_{i}} \overline{B_{i}} \overline{{ }^{t} A_{i^{\prime}}} \\
& =\overline{D_{i}} \bar{f}_{i} \overline{e_{i}} \overline{{ }^{t} A_{i^{\prime}}}=\overline{D_{i}} \overline{\tilde{e}_{i}} \overline{f_{i}} \quad \overline{{ }^{t} A_{i^{\prime}}} \\
& =\overline{D_{i}} \overline{{ }^{t} C_{i}} \overline{D_{i}} \overline{f_{i}} \bar{t}^{t} A_{i^{\prime}}=\overline{D_{i}} \overline{{ }^{t} C_{i^{\prime}}} \overline{D_{i^{\prime}}} \overline{f_{i^{\prime}}} \overline{{ }^{t} A_{i^{\prime}}} \\
& =\tilde{p}_{i i^{\prime}} \overline{D_{i^{\prime}}} \overline{f_{i^{\prime}}} \overline{{ }^{t} A_{i^{\prime}}} \text {. }
\end{aligned}
$$

In other words, if we let $\xi$ be the zero-cochain defined by $\xi_{i}=D_{i} f_{i}{ }^{t} A_{i}$, then $p_{i i^{\prime}}$ and $\tilde{p}_{i i^{\prime}}$ differ by the coboundary of $\xi_{i}$. This shows that $\left(p_{i i^{\prime}}\right)$ is well defined as a cohomology class.

To check commutativity with tensor products, note that in general if $A, B, C, D$ are matrices then $A B \otimes C D=(A \otimes C)(B \otimes D)$ so if $e={ }^{t} A \cdot B$ and $\tilde{e}={ }^{t} C \cdot D$, we get

$$
\begin{aligned}
\operatorname{im}(e) \otimes \operatorname{im}(\tilde{e}) & =\operatorname{im}(e \otimes \tilde{e})=\operatorname{im}\left(\left({ }^{t} A \otimes{ }^{t} C\right)(B \otimes D)\right) \mapsto\left(\left(\overline{B_{i}} \otimes \overline{D_{i}}\right)\left(\overline{{ }^{t} A_{i^{\prime}}} \overline{{ }^{t} C_{i^{\prime}}}\right)\right) \\
& =\left(\left(\overline{B_{i}} \overline{{ }^{t} A_{i^{\prime}}}\right) \otimes\left(\overline{D_{i}} \overline{{ }^{t} C_{i^{\prime}}}\right)\right) .
\end{aligned}
$$

We now have a well-defined map $\alpha: \operatorname{Ker}(\varphi) \rightarrow \check{H}^{1}\left(\mathscr{X}, \mathrm{GL}_{r}\right)$. We claim that $\alpha$ is injective. To see this, suppose that $P \approx \operatorname{im}(e)$ and $\tilde{P} \approx \operatorname{im}(\tilde{e})$ have the same image and write $e={ }^{t} A \cdot B$ and $\tilde{e}={ }^{t} C \cdot D$. Then there is a zero-cochain $\left(\xi_{i}\right)$ such that for 
each $i, i^{\prime}$ we have $\bar{\xi}_{i} \bar{B}_{i} \overline{{ }^{t} A_{i^{\prime}}}=\overline{D_{i}} \overline{{ }^{t} C_{i^{\prime}}} \bar{\xi}_{i^{\prime}}$. For each $i$, let $g_{i}={ }^{t} C_{i} \xi_{i} B_{i}: R_{i}^{m} \rightarrow R_{i}^{m}$. Then

$$
\begin{aligned}
\overline{g_{i}} & =\overline{{ }^{t} C_{i}} \overline{\xi_{i}} \overline{B_{i}} \\
& =\overline{{ }^{t} C_{i}} \overline{D_{i}} \overline{{ }^{t} C_{i}} \overline{\xi_{i}} \overline{B_{i}} \overline{{ }^{t} A_{i}} \overline{B_{i}} \\
& =\overline{{ }^{t} C_{i^{\prime}}} \overline{D_{i^{\prime}}} \overline{{ }^{t} C_{i}} \overline{\xi_{i}} \overline{B_{i}} \overline{{ }^{t} A_{i^{\prime}}} \overline{B_{i^{\prime}}} \\
& =\overline{{ }^{t} C_{i^{\prime}}} \overline{\xi_{i^{\prime}}} \overline{B_{i^{\prime}}}=\overline{g_{i^{\prime}}}
\end{aligned}
$$

so the $g_{i}$ define a map $g: R^{m} \rightarrow R^{m}$. We can likewise define a map $h$ by setting $h_{i}={ }^{t} A_{i} \xi_{i}^{-1} \cdot D_{i}$. Then $g h=\tilde{e}$ and $h g=e$ so $g$ and $h$ are inverse isomorphisms between $\operatorname{im}(e)$ and $\operatorname{im}(\tilde{e})$. This proves the claim.

Next we define a map $\beta: \check{H}^{1}\left(\mathcal{X}, \mathrm{GL}_{r}\right) \rightarrow \operatorname{Ker}(\varphi)$ which is inverse to $\alpha$. Given $\left(p_{i i^{\prime}}\right) \in \check{H}^{1}\left(\mathcal{X}, \mathrm{GL}_{r}\right)$, we define $\beta\left(p_{i i^{\prime}}\right)$ as the image of an idempotent matrix which we shall construct. We may assume by induction that there is an idempotent matrix $\hat{e}=\left(\hat{e}_{1}, \ldots, \hat{e}_{n-1}\right)$ over $\Gamma\left(X_{1} \cup \cdots \cup X_{n-1}\right) \subset \Pi_{i=1}^{n-1} R_{i}$ such that each $\operatorname{im}\left(e_{i}\right)$ is free and such that $\alpha(\hat{e})=\left(p_{i i^{\prime}}\right)_{i, i^{\prime}<n-1}$. Now $p_{i n}$ is invertible in $\Gamma\left(X_{i} \times_{X} X_{n}\right)$; write $q_{i n} p_{i n}=1$, and let $p_{i}$ and $q_{i}$ be arbitrary liftings of $p_{i n}$ and $q_{i n}$ to $R_{i}$. Write $p_{i} q_{i}+u_{i}=1$; squaring both sides write $p_{i} z_{i}+u_{i}^{2}=1$ where $z_{i}=q_{i} p_{i} q_{i}+2 q_{i} u_{i}$ (using the fact that $p_{i} q_{i}$ commutes with $u_{i}=1-p_{i} q_{i}$ ). Writing $e_{i}={ }^{t} \hat{A}_{i} \hat{B}_{i}$ as in the lemma, set

$$
e_{i}=\left(\begin{array}{c|c}
z_{i} p_{i} & z_{i} u_{i} \hat{B}_{i} \\
\hline{ }^{t} \hat{A}_{i} u_{i} p_{i} & { }^{t} \hat{A}_{i} u_{i}^{2} \hat{B}_{i}
\end{array}\right)=\left(\begin{array}{c}
z_{i} \\
\hline{ }^{t} \hat{A}_{i} u_{i}
\end{array}\right)\left(p_{i} \mid u_{i} \hat{B}_{i}\right)
$$

for $i<n-1$ and

$$
e_{n}=\left(\begin{array}{lllllll}
1 & & & & & 0 & \\
& \ddots & & & & & \\
& & 1 & & & & \\
& & & 0 & & & \\
0 & & & & & & 0
\end{array}\right] .
$$

Using the facts that $\hat{B}_{i}{ }^{t} \hat{A}_{i}=1$ and $p_{i} z_{i}+u_{i}^{2}=1$, it is easy to check that $e_{i}$ is idempotent.

We shall check to see that $e$ really has its entries in $R$ and that $\alpha(\operatorname{im}(e))=\left(p_{i i^{\prime}}\right)$.

To see that $e$ has its entries in $R$, we need to check that $\bar{e}_{i}=\overline{e_{i^{\prime}}}$ where "bar" denotes restriction to $X_{i} \times_{X} X_{i^{\prime}}$. By the Chinese Remainder Theorem and induction, we may assume that the $p_{i}$ have been chosen to satisfy the equation $p_{i^{\prime} i} \cdot \overline{p_{i}}=\overline{p_{i^{\prime}}}$ and consequently the $q_{i}$ can be chosen to satisfy $\bar{q}_{i} p_{i i^{\prime}}=\overline{q_{i}}$, whence $\bar{u}_{i}=1-\overline{p_{i} q_{i}}=$

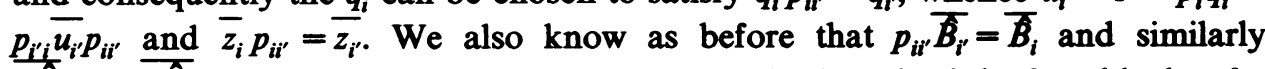
${ }^{i} \hat{A}_{i} p_{i i^{\prime}}={ }^{i} \hat{A}_{i}$. Using this information it is easy to check each of the four blocks of $e$ 
to see that $\overline{e_{i}}=\overline{e_{i^{\prime}}}$. Specifically

$$
\begin{aligned}
& \overline{z_{i^{\prime}}} \overline{p_{i^{\prime}}}=\overline{z_{i}} p_{i i^{\prime}} p_{i^{\prime} i} \overline{p_{i}}=\overline{z_{i}} \overline{p_{i}}, \\
& \overline{z_{i^{\prime}}} \overline{u_{i^{\prime}}} \overline{B_{i^{\prime}}}=\overline{z_{i}} p_{i i^{\prime}} p_{i^{\prime} i} \bar{u}_{i} p_{i i^{\prime}} p_{i^{\prime} i} \overline{B_{i}}=\overline{z_{i}} \overline{u_{i}} \overline{B_{i}} \text {, }
\end{aligned}
$$

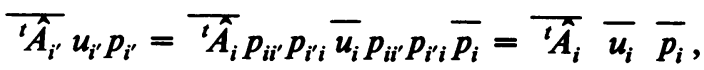

$$
\begin{aligned}
& \overline{{ }^{t} \hat{A}_{i^{\prime}}} \overline{u_{i^{\prime}}^{2}} \overline{B_{i^{\prime}}}=\overline{{ }^{t} \hat{A}_{i}} p_{i i^{\prime}} p_{i^{\prime} i} \overline{u_{i}^{2}} p_{i i^{\prime}} p_{i^{\prime} i} \overline{B_{i}}=\overline{{ }^{t} \hat{A}_{i}} \overline{u_{i}^{2}} \overline{B_{i}} \text {. }
\end{aligned}
$$

Next we compute $\alpha(\operatorname{im}(e))=\left(\xi_{i i^{\prime}}\right)$ where

$$
\begin{aligned}
\xi_{i i^{\prime}} & =\left(\overline{p_{i}} \mid \overline{u_{i}} \overline{B_{i}}\right)\left(\frac{\overline{z_{i^{\prime}}}}{\overline{{ }^{\prime} \hat{A}_{i^{\prime}}} \overline{u_{i^{\prime}}}}\right) \\
& =\overline{p_{i}} \overline{z_{i i^{\prime}}}+\overline{u_{i}} \overline{\bar{B}_{i}} \overline{{ }^{i} \hat{A}_{i i^{\prime}}} \overline{u_{i^{\prime}}}=\overline{p_{i} z_{i}} p_{i i^{\prime}}+\overline{u_{i}} p_{i i^{\prime}} p_{i^{\prime} i} \overline{u_{i}} p_{i i^{\prime}} \\
& =\left(\overline{p_{i} z_{i}}+\overline{u_{i}}\right) p_{i i^{\prime}}=p_{i i^{\prime}}
\end{aligned}
$$

when $i, i^{\prime}<n-1$. Also

$$
\xi_{\text {in }}=\left(\begin{array}{ll}
\overline{p_{i}} \mid \overline{u_{i}} & \overline{B_{i}}
\end{array}\right)\left(\frac{I}{0}\right)=\overline{p_{i}}=p_{i n} .
$$

This (combined with the fact that $\alpha$ is one-one) shows that $\beta$ is well-defined and inverse to $\alpha$, proving the theorem. Q.E.D.

Remark. R. G. Swan has pointed out to me via the following example that the condition $X=$ ind $\lim X_{i}$ is really necessary: Let $X_{1}=\operatorname{Spec}(k[X, Y, Z] / X Y Z)$ and let $X_{2}=\operatorname{Spec}(k[X, Y] / X Y(X-Y))$. Then the coverings $\mathcal{X}_{1}$ and $\mathscr{X}_{2}$ of $X_{1}$ and $X_{2}$ by their irreducible components are isomorphic, and $\operatorname{Pic}\left(X_{1}\right)=$ $H^{1}\left(\mathscr{X}_{1}, \Theta_{X_{1}^{*}}^{*}\right)=0$ by the theorem, whereas $\operatorname{Pic}\left(X_{2}\right)=k \neq H^{1}\left(\mathscr{X}_{2}, \theta_{X_{2}^{*}}^{*}\right)$.

In everything that follows, $X$ will denote an affine scheme.

COROLLARY 2.2. If $\left\{X_{i}\right\}$ has cardinality $n$ and $\xi$ is a vector bundle of rank $r$ such that $\left.\xi\right|_{X_{i}}$ is trivial for each $i$, then $\xi$ can be generated by $n r$ sections.

Proof. The matrix $e$ constructed in the proof of the theorem is an $n r \times n r$ matrix. Q.E.D.

In some circumstances, the proof of Theorem 2.1 gives much better information about generators:

Definition. $A$ cochain $\left(p_{i i^{\prime}}\right)$ is special if there is an index $i_{0}$ such that whenever $i \neq i_{0}$ and $i^{\prime} \neq i_{0}$ it follows that $p_{i i^{\prime}}=1$.

LEMMA. In the situation of the theorem, if $P \in \operatorname{ker}(\varphi)$ is represented by a special cocycle then $P$ can be generated by $2 r$ sections (where $r$ is the rank of $P$ ).

Proof. Let $\left(p_{i i^{\prime}}\right)$ represent $P$ and let $i_{0}$ be as in the definition of special. For each $i$, write $p_{i i_{0}} \cdot q_{i i_{0}}=1$ and let $p_{i}$ and $q_{i}$ be arbitrary liftings of $p_{i i_{0}}$ and $q_{i i_{0}}$ to $R_{i}$. Write $p_{i} q_{i}+u_{i}=1$ so $p_{i} z_{i}+u_{i}^{2}=1$ where $z_{i}=q_{i} p_{i} q_{i}+2 q_{i} u_{i}$. For each $i \neq i_{0}$, let

$$
e_{i}=\left(\begin{array}{c|c}
z_{i} p_{i} & z_{i} u_{i} \\
\hline u_{i} p_{i} & u_{i}^{2}
\end{array}\right)
$$


and let

$$
e_{i_{0}}=\left(\begin{array}{l|l}
I & 0 \\
\hline 0 & 0
\end{array}\right)
$$

Having chosen the $p_{i}$ and $q_{i}$ carefully as in the proof of the theorem, we may assume $\overline{p_{i}}=\overline{p_{i^{\prime}}}$ (and consequently $\bar{q}_{i}=\overline{q_{i^{\prime}}}, \overline{u_{i}}=\overline{u_{i^{\prime}}}$ ) for each $i, i^{\prime}$ distinct from $i_{0}$. Then $\alpha(\operatorname{im} e)=\left(\xi_{i i^{\prime}}\right)$ where for $i, i^{\prime} \neq i_{0}$ we have $\xi_{i i^{\prime}}=\overline{p_{i} z_{i^{\prime}}}+\overline{u_{i} u_{i^{\prime}}}=\overline{p_{i} z_{i}}+\overline{u_{i}^{2}}=1$, and where $\xi_{\text {in }}=\bar{p}_{i}=p_{i n}$. That is, $\left(\xi_{i i^{\prime}}\right)=\left(p_{i i^{\prime}}\right)$. But $\alpha$ : Ker $\varphi \rightarrow \check{H}^{1}\left(\mathcal{X}, \mathrm{GL}_{r}\right)$ is injective so $P \approx \operatorname{im}(e)$. Thus $P$ can be generated by $2 r$ sections. Q.E.D.

REMARK. As a consequence of this lemma, any line bundle which becomes trivial on each $X_{i}$ and whose cocycle is a product of special cocycles is a tensor product of line bundles which can be generated by two sections. In particular, when the special cocycles generate the group of cocycles, every line bundle can be written as such a tensor product. The special cochains always generate the group of cochains, so we get

COROllary 2.3. If whenever $i, i^{\prime}, i^{\prime \prime}$ are all distinct it follows that $X_{i} \times_{X} X_{i^{\prime}}$ $\times_{X} X_{i^{\prime \prime}}=\varnothing$, then every line bundle which becomes trivial on each $X_{i}$ is a tensor product of line bundles which can be generated by two sections.

Proof. In this case the group of cochains is the group of cocycles, so we are done by the remark above. Q.E.D.

We can strengthen this:

COROllary 2.4. Suppose that for every three distinct indices $i, i^{\prime}, i^{\prime \prime}$ we have either $X_{i} \times_{X} X_{i^{\prime}} \times_{X} X_{i^{\prime \prime}}=\varnothing$ or $X_{i} \times_{X} X_{i^{\prime}} \times_{X} X_{i^{\prime \prime}}=X_{i} \times_{X} X_{i^{\prime}}$. Then every line bundle on $X$ which becomes trivial on each $X_{i}$ is a tensor product $L_{1} \otimes \cdots \otimes L_{n-1}$ where each $L_{i}$ can be generated by two sections.

Proof. Let $L$ be a line bundle and set $\alpha(L)=\left(p_{i i^{\prime}}\right)$. For each index $i_{0}$ let $\left(p_{i i^{\prime}}^{i_{0}}\right)$ be the cocycle defined by

$$
\begin{aligned}
& p_{i_{0^{j_{0}}}}^{i_{0}}=1, \\
& p_{i_{0^{j^{\prime}}}}^{i_{0}}=p_{i_{\alpha_{0}}} \text { where } l_{0}=l_{0}\left(i_{0}, i^{\prime}\right)=\max \left\{l \mid X_{i_{0}} \times_{X} X_{i^{\prime}} \times_{X} X_{l} \neq \varnothing\right\}, \\
& p_{i i_{0}}^{i_{0}}=\left(p_{i_{0}}^{i_{0}}\right)-1, \\
& p_{i i^{\prime}}^{i_{0}}=1 \text { if } i, i^{\prime} \neq i_{0} .
\end{aligned}
$$

To see that this is a cocycle, suppose that $X_{i_{0}} \times{ }_{X} X_{i} \times{ }_{X} X_{i^{\prime}} \neq \varnothing$. Then $X_{i_{0}} \times_{X} X_{i}$ $=X_{i_{0}} \times_{X} X_{i^{\prime}}$ so $l_{0}\left(i_{0}, i\right)=l_{0}\left(i_{0}, i^{\prime}\right)$, whence $p_{i i_{0}}^{i_{0}} p_{i_{0} i^{\prime}}^{i_{0}}=p_{l_{0_{0} j_{0}}} p_{i_{0} d_{0}}=1=p_{i i^{\prime}}$ (assuming $\left.i, i^{\prime} \neq i_{0}\right)$. One checks the other triple intersections similarly.

Now for fixed $i, i^{\prime}$,

$$
\prod_{i_{0}=1}^{n} p_{i i^{\prime}}^{i_{9}}=p_{i i^{\prime}}^{i} p_{i i^{\prime}}^{i^{\prime}}=p_{i l_{0}\left(i, i^{\prime}\right)} \cdot p_{l_{0}\left(i, i^{\prime}\right) i^{\prime}}=p_{i i^{\prime}},
$$

so

$$
\left(p_{i i^{\prime}}\right)=\prod_{i=1}^{n}\left(p_{i i^{\prime}}^{i_{0}}\right)
$$

Thus if $L_{i_{0}}$ is the line bundle defined by $\left(p_{i i}^{i q}\right)$, we have $L=L_{1} \otimes \cdots \otimes L_{n}$. But each $\left(p_{i i}^{i}\right)$ is special, so each $L_{i}$ can be generated by two sections. Moreover, $L_{n}$ is 
trivial since $p_{i i^{\prime}}^{n}=1$ for all $i, i^{\prime}$. Therefore $L=L_{1} \otimes \cdots \otimes L_{n-1}$ where each $L_{i}$ can be generated by two sections. Q.E.D.

REMARK. The proof of the above corollary is still valid if we define $p_{i i^{\prime}}^{i_{0}}$ separately on each connected component $Y$ of $X_{i} \times_{X} X_{i^{\prime}}$ by

$$
\left.p_{i_{0^{\prime}}}^{i_{0^{\prime}}}\right|_{Y}=p_{i_{0_{0}}} \quad \text { where } l_{0}=l_{0}\left(i_{0}, Y\right)=\max \left\{l \mid Y \times_{X} X_{l} \neq \varnothing\right\} .
$$

This gives the stronger result:

COROLlaRY 2.5. Suppose that for every three indices $i, i^{\prime}, i^{\prime \prime}$ it is the case that each connected component of $X_{i} \times_{X} X_{i^{\prime}} \times_{X} X_{i^{\prime \prime}}$ is a connected component of $X_{i} \times_{X} X_{i^{\prime}}$. Then every line bundle over $X$ which becomes trivial on each $X_{i}$ can be written as a tensor product $L_{1} \otimes \cdots \otimes L_{n-1}$ where each $L_{i}$ can be generated by two sections.

An immediate consequence of this is

Corollary 2.6. Suppose that all but at most one of the $X_{i}$ are one-dimensional. Then every line bundle over $X$ which becomes trivial on each $X_{i}$ can be written as a tensor product $L_{1} \otimes \cdots \otimes L_{n-1}$ where each $L_{i}$ can be generated by two sections.

As another corollary to the proof of Theorem 2.1, we get a partial generalization of the well-known fact that if $I$ is any nil ideal in $R$ then any projective module over $R / I$ is extended from $R$.

Corollary 2.7. Suppose that there exists an index $i_{0}$ such that each $X_{i} \times{ }_{X} X_{i_{0}}$ is defined by a nil ideal in $\Gamma\left(X_{i}\right)$. Then any vector bundle which is trivial on each $X_{i}$ is trivial.

Proof. Assume $i_{0}=n$. Then, in the proof of Theorem 2.1, $u_{i}$ is in the ideal defining $\Gamma\left(X_{i} \times_{X} X_{n}\right)$ in $\Gamma\left(X_{i}\right)$. Write $u_{i}^{k_{i}}=0$ and let $k$ be greater than $\max _{i} k_{i}$. Writing $p_{i} z_{i}=u_{i}^{2}=1$ as in the theorem, raise each side to the $k$ to get $p_{i} w_{i}=1$. Then for each $i, i^{\prime}, p_{i i^{\prime}}=p_{i} w_{i^{\prime}}$, so $\left(p_{i i^{\prime}}\right)$ is a coboundary. Q.E.D.

III. Stability. We continue to use the notation of the previous section. In addition, $E_{r}$ will denote the sheaf of $r \times r$ elementary matrices on $X$ and $E$ will denote $\cup_{r=1}^{\infty} E_{r}$ (where $E_{r}$ is contained in $E_{r+1}$ in the usual way).

THEOREM 3.1. Let $L$ be the kernel of the restriction map

$$
\tilde{K}_{0}(X) \rightarrow \bigoplus_{i=1}^{n} \tilde{K}_{0}\left(X_{i}\right)
$$

Then there is a natural exact sequence

$$
H^{1}(\mathcal{X}, E) \rightarrow L \stackrel{\hat{\alpha}}{\rightarrow} H^{1}\left(\mathscr{X}, K_{1}\right) .
$$

Proof. We use the notation from the proof of Theorem 2.1. Given $([P]-[F]) \in$ $L$ with $F$ free and $P$ of rank $r$, let $\hat{\alpha}(P)$ be the image of $\alpha(P)$ under the map $H^{1}\left(\mathcal{X}, \mathrm{GL}_{r}\right) \rightarrow H^{1}\left(\mathcal{X}, K_{1}\right)$.

This is well defined: if $[P]-[F]=[Q]-\left[F^{\prime}\right]$ with $F$ and $F^{\prime}$ free, then $P \oplus F^{\prime \prime}$ $\approx Q \oplus F^{\prime \prime \prime}$ for appropriate free modules $F^{\prime \prime}, F^{\prime \prime \prime}$. The claim that $\hat{\alpha}$ is well defined now follows from the fact that $\hat{\alpha}(P)=\hat{\alpha}\left(P \oplus F^{\prime \prime}\right)=\hat{\alpha}\left(Q \oplus F^{\prime \prime \prime}\right)=\hat{\alpha}(Q)$. 
This is a group homomorphism: $\hat{\alpha}\left([P]-[F]+[Q]-\left[F^{\prime}\right]\right)$ is the image of

$$
\alpha(P \oplus Q)=\left(\begin{array}{c|c}
\alpha(P) & 0 \\
\hline 0 & \alpha(Q)
\end{array}\right),
$$

which, by Whitehead's Lemma, is equal in $K_{1}$ to $\alpha(P) \alpha(Q)$.

Finally defining a map $H^{1}(\mathcal{X}, E) \rightarrow L$ by composing the natural map $H^{1}(\mathcal{X}, E)$ $\rightarrow H^{1}(\mathscr{X}, \mathrm{GL})$ with the map $H^{1}(\mathscr{X}, \mathrm{GL}) \rightarrow L$ given by Theorem 2.1 , we see that if $\hat{\alpha}(P)=0$, then $\alpha(P)$ is stably elementary and so comes from $H^{1}(\mathcal{X}, E)$. Q.E.D.

Note. The above proof simply amounts to noting that the natural map $H^{1}(\mathcal{X}, \mathrm{GL}) \rightarrow \tilde{K}_{0}(X)$ factors through $L$ and that the vertical arrow in the diagram

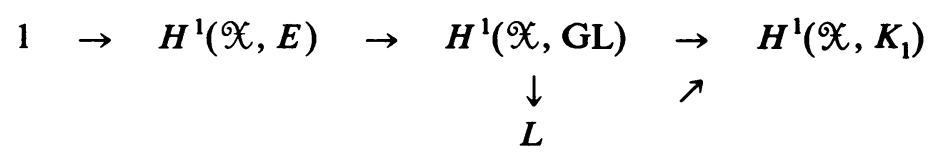

is onto.

Corollary 3.2. If $H^{1}(\mathcal{X}, E)=0$ then $L$ maps injectively to $H^{1}\left(\mathcal{X}, K_{1}\right)$.

As an example, when $\mathfrak{X}$ satisfies the hypothesis of Corollary 2.5 an argument using the fact that $E_{r}$ is "flasque" with respect to the covering $\mathcal{X}$ shows that $H^{1}\left(\mathfrak{X}, E_{r}\right)=0$ for each $r$, so that Corollary 3.2 applies.

COROLlary 3.3. Suppose that $X$ satisfies the hypotheses of Corollary 2.5 and that $\tilde{K}_{0}\left(X_{i}\right)=0$ for each $i$. Suppose also that $H^{1}\left(X, S K_{1}\right)=0$. Then $\tilde{K}_{0}(X)=\operatorname{Pic}(X)$.

Proof. We have $\tilde{K}_{0}(X) \hookrightarrow H^{1}\left(\mathcal{X}, K_{1}\right)=H^{1}\left(\mathcal{X}, \mathrm{GL}_{1}\right) \oplus H^{1}\left(\mathcal{X}, S K_{1}\right)=\operatorname{Pic}(X)$; it is easy to check that this is just the determinant map, which is onto. Q.E.D.

EXAMPLE 3.4. Let $R$ be a regular ring such that $\tilde{K}_{0}(R)=S K_{1}(R)=0$. Suppose that each $X_{i}$ and each $X_{i} \times_{X} X_{j}$ is an affine space over $R$, and that the hypothesis of Corollary 2.5 is satisfied. Then $\tilde{K}_{0}(X)=\operatorname{Pic}(X)$.

\section{Applications.}

A. Coverings and triangulations. Suppose that either $K=\mathbf{R}, k=1, R=\mathbf{Z}_{2}$, or $K=\mathbf{C}, k=2$, and $R=\mathbf{Z}$. Let $X$ be a compact space, and $\xi$ a $K$-line bundle on $X$. Let $c \in H^{k}(X, R)$ be the first Stiefel-Whitney class of $\xi$ if $K=\mathbf{R}$, or the first Chern class of $\xi$ if $K=\mathbf{C}$. The following argument must be well known, but I have not been able to find a specific reference:

LemMa. If $\xi$ can be generated by $n$ sections then the $n$-fold cup product $c^{n} \in$ $H^{n k}(X, R)$ is zero.

Proof. Let $\varphi_{\xi}: X \rightarrow K \mathrm{P}^{\infty}$ be the classifying map for $\xi$. The $n$ sections define a factorization

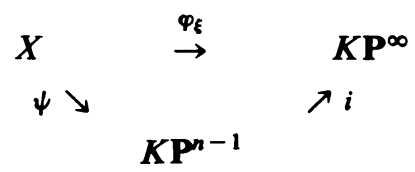

so that $c=\psi^{*} \hat{c}$ for some $\hat{c} \in H^{k}\left(K P^{\infty}, R\right)$. But $H^{n k}\left(K P^{n-1}, R\right)=0$. Q.E.D. 
THEOREM 4.1. Let $X$ be any compact Hausdorff space which can be covered by finitely many closed subspaces $X_{1}, \ldots, X_{n}$ such that $H^{k}\left(X_{i}, R\right)=0$ for each $i$. Suppose that for any distinct $i, i^{\prime}, i^{\prime \prime}$, every connected component of $X_{i} \cap X_{i^{\prime}} \cap X_{i^{*}}$ is a connected component of $X_{i} \cap X_{i^{\prime}}$. Then every $K$-line bundle over $X$ can be written as a tensor product $L_{1} \otimes \cdots \otimes L_{n-1}$ where each $L_{i}$ can be generated by two sections.

Proof. By Swan's Theorem [11], every vector bundle on $X$ is a pullback of a vector bundle over $\hat{X}=\operatorname{Spec}(C(X, K))$ under the natural inclusion $X \hookrightarrow \hat{X}$. Because $X$ is normal, each $X_{i}$ is the zero set of some continuous function, so $\left\{\hat{X}_{i}\right\}_{i=1, \ldots, n}$ is a closed covering of $\hat{X}$. Each $\operatorname{Pic}\left(\hat{X}_{i}\right)$ is trivial because we assumed $H^{k}\left(X_{i}, R\right)=0$, so, by Corollary 2.5 , every line bundle over $\hat{X}$ is of the required form, whence every line bundle over $X$ is also. Q.E.D.

Corollary 4.2. If $X$ is a space which can be covered as in the statement of the theorem, then $H^{k}(X, R)$ is generated as a group by elements $c$ satisfying $c \cap c=0$.

Corollary 4.3. Neither $\mathbf{R P}^{n}$ nor $\mathbf{C P}^{n}$ can be covered as in the statement of the theorem, for $n>2$.

COROllary 4.4. For $n>2$, any triangulation of $\mathbf{R P}^{n}$ or $\mathbf{C P}^{n}$ includes three $n$-simplices $A, B, C$ such that $\varnothing \neq A \cap B \cap C \neq A \cap B$.

Proof. By "fattening things up" where necessary, we may assume that every simplex is a face of an $n$-simplex, so the $n$-simplices cover. Q.E.D.

RemarK. Let $X$ be any space for which Swan's Theorem holds. Set

$L(X)=$ the Lusternik-Schnirelman category of $X$ (i.e., the smallest number of closed contractible sets needed to cover $X)$.

$V(X)=$ the maximum, as $\xi$ varies over all $K$-line bundles over $X$, of the minimal number of sections necessary to generate $\xi$.

$C(X)=$ cuplong $(X)=$ the largest integer $n$ such that for some field $F$ and elements $c_{i} \in H^{k_{1}}(X, F), k_{i}>0,1<i<n$, we have $c_{1} \cup \cdots \cup c_{n} \neq 0$.

$D(X)=$ the largest integer $n$ such that there exists $c \in H^{k}(X, R)$ with $c^{n} \neq 0$.

Then the sorts of arguments made above show that $L(X)>V(X)>D(X)+1$. It is known [9] that $L(X)>C(X)+1$ and obvious that $C(X)>D(X)$, so we have an array

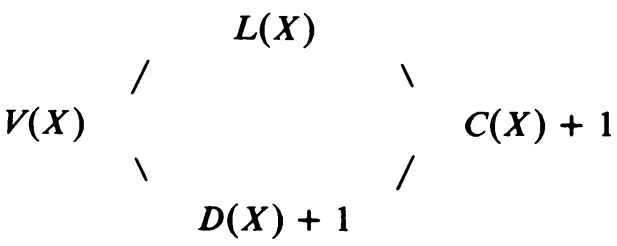

where each number is greater than those displayed below it.

We also have

THEOREM 4.5. Let $X$ be any compact Hausdorff space which can be covered by finitely many closed subspaces $X_{1}, \ldots, X_{n}$ such that $\tilde{K}_{0}\left(X_{i}\right)=0$ for each $i$. Suppose that for any distinct $i, i^{\prime}, i^{\prime \prime}$, every connected component of $X_{i} \cap X_{i^{\prime}} \cap X_{i^{\prime \prime}}$ is a connected component of $X_{i} \cap X_{i^{\prime}}$. Then $\tilde{K}_{0}(X)=\operatorname{Pic}(X)$ (where $K_{0}$ and Pic refer to the topological $K_{0}$ and Picard groups). 
Proof. This can be proved just as Theorem 4.1 is proved, invoking Corollary 3.3 instead of Corollary 2.5.

B. Cohomology of complex varieties.

THEOREM 4.6. Let $X$ be a complex affine variety with irreducible components $X_{1}, \ldots, X_{n}$ satisfying the hypotheses of Theorem 2.1. Suppose that there is a group $G$ such that for each $i, j$ the group of units on $X_{i} \cap X_{j}$ is isomorphic to $G$. Suppose also that for each $i, \operatorname{Pic}\left(X_{i}\right)=0=H^{1}\left(X_{i}, G\right)$. (For example, the $X_{i}$ could all be nonsingular and contractible-see Gurjar [4].) Then $\operatorname{Pic}(X)=H^{1}(X, G)$.

Proof. Consider the sequences

$$
\begin{aligned}
& \begin{array}{c}
0 \rightarrow \check{H}^{1}\left(X, \Theta_{X}^{*}\right) \\
\|
\end{array} \rightarrow \operatorname{Pic}(X) \rightarrow \bigoplus_{i=1}^{n} \operatorname{Pic}\left(X_{i}\right)=0 \\
& 0 \rightarrow \check{H}^{1}(\mathcal{X}, G) \quad \rightarrow \quad H^{1}(X, G) \rightarrow \bigoplus_{i=1}^{n} H^{1}(X, G)=0
\end{aligned}
$$

Here the top row is the exact sequence from Theorem 2.1, and the bottom row is the standard exact sequence from topology [1] (actually, this sequence requires the $X_{i}$ to be open, but this can be arranged by embedding $X$ in $\mathbf{C}^{N}$ and then replacing $X$ and the $X_{i}$ by sufficiently small open neighborhoods of themselves, which does not affect the nerve of $\mathcal{X}$ or the singular cohomology groups). The indicated equalities hold by assumption and the theorem follows immediately. Q.E.D.

ReMark. This does not follow directly from Theorem 1.5, as that would require an assumption about $X_{i} \cap\left(\cup_{j \in S} X_{j}\right)$ for various subsets $S \subset\{1, \ldots, n\}$.

THEOREM 4.7. Suppose that $X$ is a complex affine variety and that there is a group $G$ such that for each $i, j$ we have $K_{1}\left(X_{i} \times_{X} X_{j}\right)=G$. Suppose also that for each $i$ there is an injection $\tilde{K}_{0}\left(X_{i}\right) \hookrightarrow H^{1}\left(X_{i}, G\right)$, and that the hypothesis of Corollary 2.5 is satisfied. Then there is an injection $\tilde{K}_{0}(X) \hookrightarrow H^{1}(X, G)$.

Proof. Invoke Corollary 3.3.

C. Torsion in Picard groups.

THEOREM 4.8. Let $K$ be an affine scheme with irreducible components $X_{1}, \ldots, X_{n}$. Suppose that there is a group $G \subset \mathcal{O}^{*}$ such that for each $i, j$ the units on $X_{i} \times_{X} X_{j}$ are isomorphic to $G$, and suppose also that $\operatorname{Pic}\left(X_{i}\right)=0$ for each $i$. Let $k>0$ be an integer. Then:

(1) If $G$ has no $k$-torsion, then neither does $\operatorname{Pic}(X)$.

(2) If $G$ is a $k$-torsion group, then so is $\operatorname{Pic}(X)$.

Proof. Let $\bar{X}=X \otimes_{\mathrm{z}} \mathrm{C}$ and let $\bar{X}=\left\{\bar{X}_{1}, \ldots, \bar{X}_{n}\right\}$. Then by Theorem 2.1, $\operatorname{Pic}(X)=\check{H}^{1}\left(\mathcal{X}, \Theta^{*}\right)$. By assumption this is the same as $\check{H}^{1}(\mathcal{X}, G)$, which it is easy to see is the same as $\check{H}^{1}(\overline{\mathcal{X}}, G)$. But this is a subgroup of $H^{1}(\bar{X}, G)$ as in the proof of Theorem 4.6, and it is well known [1] that $H^{1}(\bar{X}, G)=$ $\operatorname{Hom}_{\mathrm{Z}}\left(H_{1}(\bar{X}, \mathbf{Z}), G\right)$ which completes the proof. Q.E.D. 


\section{REFERENCES}

1. A. Dold, Lectures on algebraic topology, Springer-Verlag, Berlin, 1972.

2. E. G. Evans, Jr., Projective modules as fiber bundles, Proc. Amer. Math. Soc. 27 (1971), 623-626.

3. R. Fossum, Vector bundles over spheres are algebraic, Invent. Math. 8 (1969), 222-225.

4. R. Gurjar, Thesis, University of Chicago, 1979.

5. K. Lonsted, An algebraization of vector bundles on compact manifolds, J. Pure Appl. Algebra 2 (1972), 193-207.

6. __ Vector bundles over finite $C W$ complexes are algebraic, Proc. Amer. Math. Soc. 105 (1962), 264-277.

7. J. Milnor, Introduction to algebraic K-theory, Princeton Univ. Press, Princeton, N. J., 1971.

8. C. Mulvey, A generalization of Swan's Theorem, Math. Z. 151 (1976), 57-70.

9. R. S. Palais, Lusternik-Schnirelman theory on Banach manifolds, Topology 5 (1966), 115-132.

10. J.-P. Serre, Faisceaux algébriques cohérents, Ann. of Math. (2) 6 (1955), 197-278.

11. R. G. Swan, Vector bundles and projective modules, Trans. Amer. Math. Soc. 105 (1962), 264-277.

12. T_ Topological examples of projective modules, Trans. Amer. Math. Soc. 230 (1977), 201-234.

13. R. G. Swan and J. Towber, A class of projective modules which are nearly free, J. Algebra 36 (1975), 427-434.

Department of Economics, University of Chicago, Chicago, Illinois 60637 